\title{
Prototype development for real-time epilepsy seizures detector using three parameters
}

\author{
Joyce S. Y. Sia ${ }^{1}$, Lai Z. Huan ${ }^{1}$, Lam S. Kit ${ }^{1}$ and Liong C. Ling ${ }^{1}$, Ghazali N. Effiyana ${ }^{1, *}$ \\ ${ }^{1}$ Faculty of Electrical Engineering, Universiti Teknologi Malaysia, 81310 Johor Bahru, Johor \\ Malaysia \\ ${ }^{2}$ Advanced Telecommunication Technology Research Group, Faculty of Electrical Engineering, \\ Universiti Teknologi Malaysia, 81310 Johor Bahru, Johor Malaysia
}

\begin{abstract}
This paper proposes a prototype for real-time epilepsy seizures detection using skin conductance, temperature and sense movement. This proposed work is expected to help epilepsy patients to receive immediate help from the people around when seizures happen. This prototype is wearable and developed using Arduino Nano, Galvanic Skin Response (GSR) sensor, accelerometer, temperature sensor and pulse sensor. Epilepsy patients can wear this prototype just like a watch. The prototype is connected to the mobile application via Bluetooth and can alert the people around by buzzing alarm as well as sending text message to the doctor or family member. Details development and results are discussed in this paper.
\end{abstract}

\section{Introduction}

The ubiquitous computing and mobile communications create a smart technology platform for healthcare. This emerging trend provides advanced medicine and clinical care as well as bring benefit to individual patients and society as whole. Epilepsy seizures as a challenge in specialty care due to the problem of lack of neurologists and no accurate therapy for each type of epilepsy [1].

Epilepsy is abnormal of central nervous system in which the brain's electrical systems that are characterized by a disorder tendency to cause recurrent seizures. The thought, action and feeling of patients become confused and uncontrollable when the communication between nerve cells of brain is disrupted [2]. The patient temporary lapses of consciousness and loss their body controls suddenly may cause severe or subtle injuries. The frequency of seizures happen is unpredictable [3]. Therefore, seizures first aid plays a critical role to reduce the danger toward the patients.

Most type of epilepsy seizures can be classified into two main categories which are generalized seizures and focal seizures [4]. Focal seizures happen when certain part of the brain in abnormal condition which means only part of the body controlled by that part of brain will be affected. The severity of focal seizures depends on the area of seizures, the part of brain where seizures happen and the particular function of that part of brain [1].

*Corresponding author: nurzal@utm.my 
However, generalized seizures start deep of the brain involve the entire brain may cause disoriented of whole body. The generalized seizures can be divided into motor or nonmotor seizures. The cause of generalized seizures is presumed to be genetic [2].

Particular symptoms can be the indicator and sign of epilepsy seizures happen. Neurologists are able to provide more information to the patient about the reasons of seizures happen when a person is diagnosed with a particular syndrome. Symptoms of epilepsy seizures include staring and blinking, jerking movements, loss of muscle tone and stiffening of limbs [4]. When both sides of brain are involved, symptoms include rhythmic and full-body jerking. Delay detection cause premature death of epilepsy seizures' patients.

Therefore, for this project, a device is proposed to detect the happens of epilepsy seizures. Practical suggestions are provided where this epilepsy seizures detector is developed to achieve timely detection to prevent premature death. This device will detect the skin conductive, orientation and temperature of patients in the process to detect whether the epilepsy seizures is happened or not.

\section{Prototype Development}

The basic requirement of this project is to produce a wearable device which is small in size and portable so that user is easily to wear the epilepsy seizure detector to alert the surrounding people when seizure is happened. The design of this hardware is almost same to the wearable products that we can see in the market such as Apple watch and other type of smart watches. After interviewing a medical doctor from Pusat Kesihatan UTM (PKU), the conclusion that can be made is the obstacles facing by the patients of epilepsy seizure nowadays is lack of suitable approaches which can give them first aids when seizures happen. Most of the fatalities cause by seizures is because of the delay treatment. The pain point that is solved is listed in Table 1 .

Table 1. Pain points and needs

\begin{tabular}{|l|l|}
\hline \multicolumn{1}{|c|}{ Pain Point } & \multicolumn{1}{c|}{ Needs } \\
\hline $\begin{array}{l}\text { The seizure can happen regardless } \\
\text { time and places. }\end{array}$ & $\begin{array}{l}\text { Alert the surrounding people and } \\
\text { provide some first aids knowledge about } \\
\text { seizures to public. }\end{array}$ \\
\hline $\begin{array}{l}\text { Severe symptoms may cause } \\
\text { fatal. }\end{array}$ & $\begin{array}{l}\text { Monitor health progress to get the } \\
\text { condition of patient immediately. }\end{array}$ \\
\hline $\begin{array}{l}\text { Seizure may cause physical } \\
\text { injuries. }\end{array}$ & $\begin{array}{l}\text { Alongside with the patient in order to } \\
\text { provide immediate assistance or help to } \\
\text { the patients. }\end{array}$ \\
\hline
\end{tabular}

The goal of this project is to provide instant help to the epilepsy seizures patients as well as to reduce the cases of fatalities cause by prolonger treatment. However, the current technology and lack of information from professional specialist in neurology become the limitation for the perfection of this prototype. 
Figure 1 illustrates the general architecture of this project.

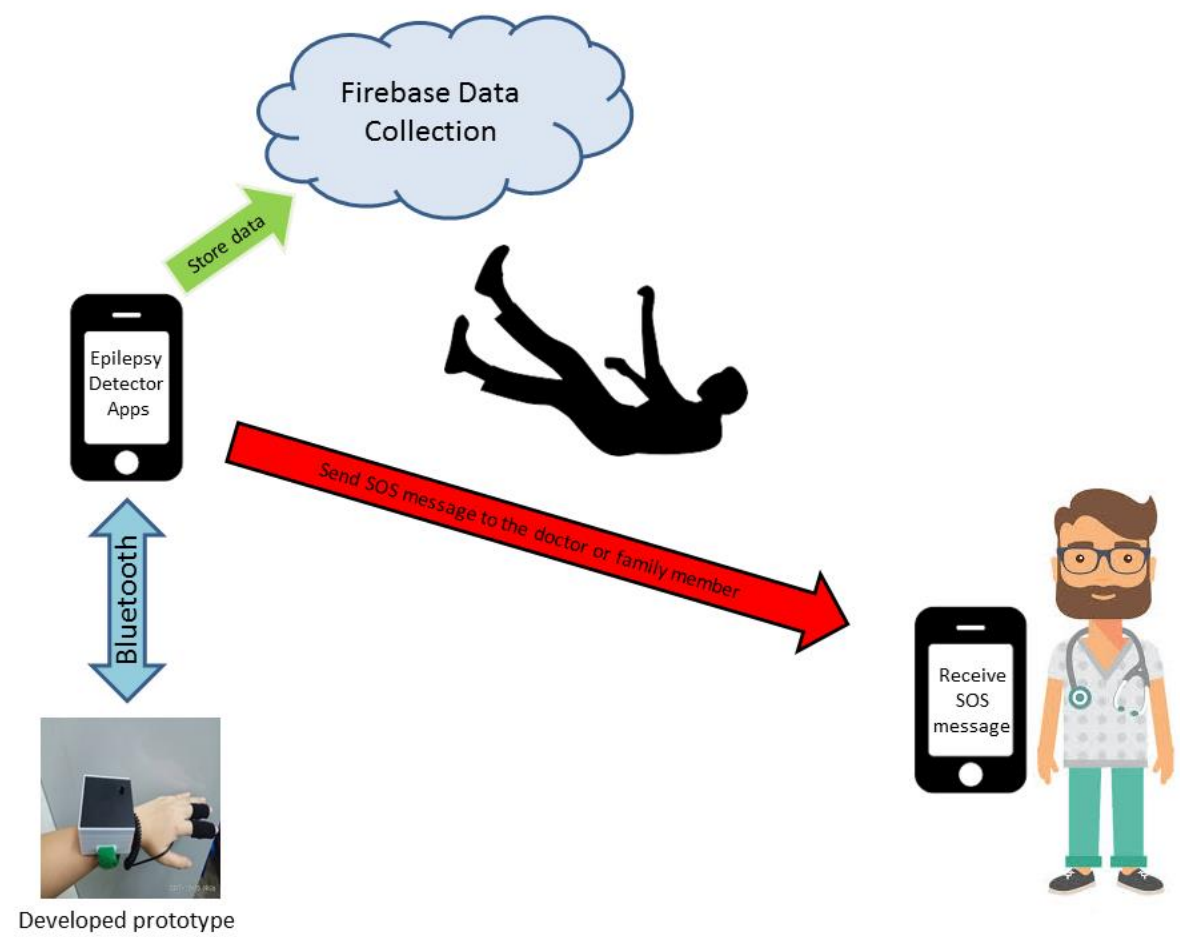

Fig. 1. General architecture

Figure 1 shows the developed prototype is connected to the developed epilepsy seizure detector apps via Bluetooth. The developed apps stores all the data from the sensors in Firebase database. SOS message will be send to the doctor or family member in real-time when seizure happens. At the same time, patient's phone will turn on the buzzer to alert people around the patient. The first aid instruction will be shown in the patient's phone via the developed apps.

The proposed epilepsy seizures detector will determine the happens of seizures by using three references which are skin conductance, temperature and sense movement of the patient. A Galvanic Skin Response (GSR) sensor is used to detect the skin conductance of the patient because the muscle of the patient will cramp when seizures happen. The sense of movement of the patient is determined by using accelerometer as a detection of falling of patients. These are the two main sensors for the detection of epilepsy seizures and it is supported by a temperature sensor. As the temperature of patient increase from normal temperature, it has higher possibility happens of seizures. All of these sensors will be connected to the Arduino Nano board for controlling. It is dangerous when the patient is not accompanied by family member as seizures happens. Thus, a Bluetooth is implemented in the system to trigger the developed epilepsy seizure detector apps to send the emergency message to the preliminary setting emergency contact number. The contact number can be doctor's patient or family member. At this current time, only one number can be inserted to set as the emergency number that can receive the emergency message. Figure 2 shows the flow chart of the system. 


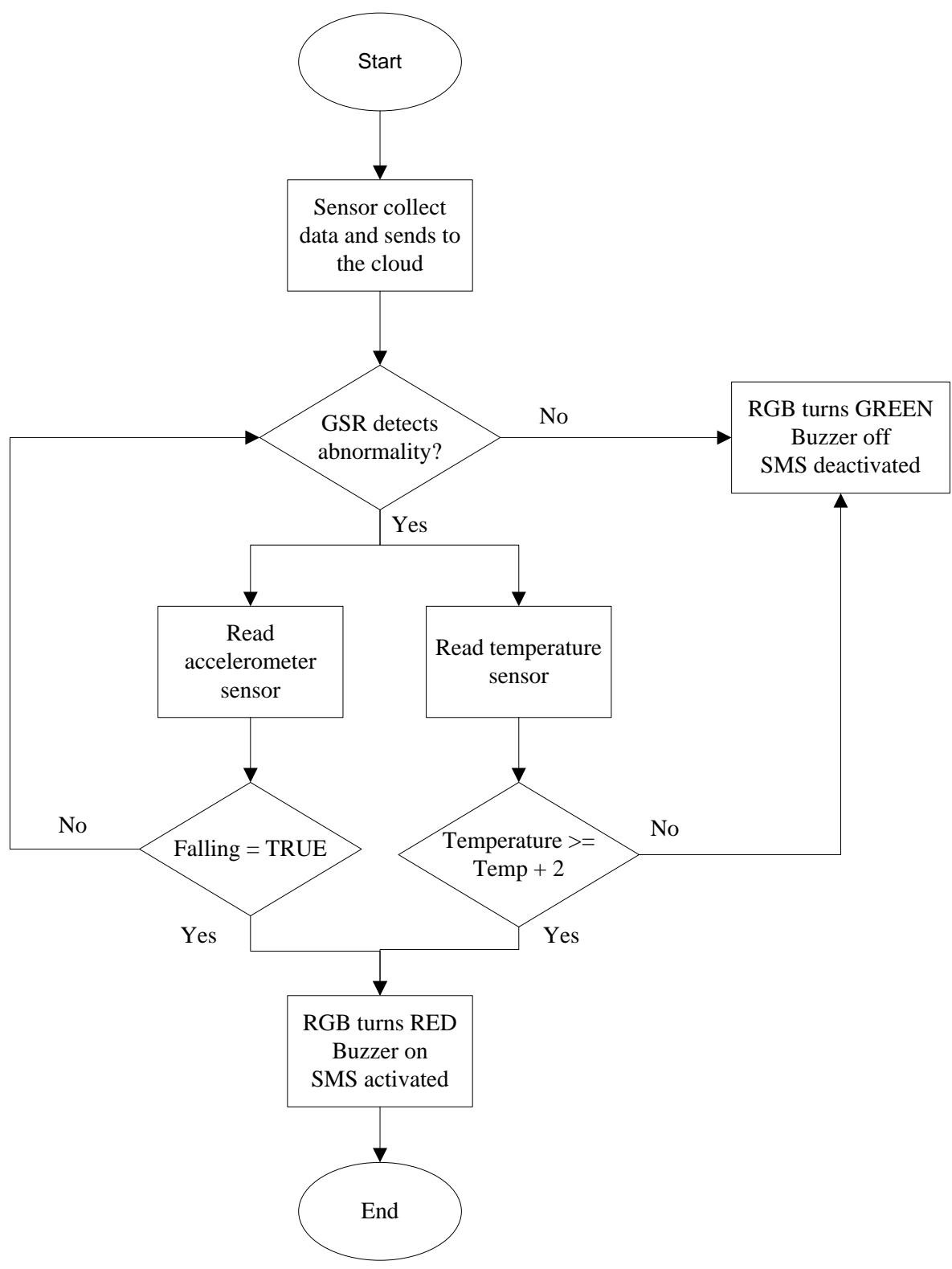

Fig. 2. Flowchart of the system's operation

\section{System Operation}

The coding was reloaded to the Arduino Nano board to ensure all the readings taken were newly generated. Two GSR sensor electrodes were wore on two fingers of patient for detecting skin conductance purpose. First-time usage button was pressed until the initially red in colour Red, Green, Blue (RGB) LED is turned to green as shown in Figure 3. 


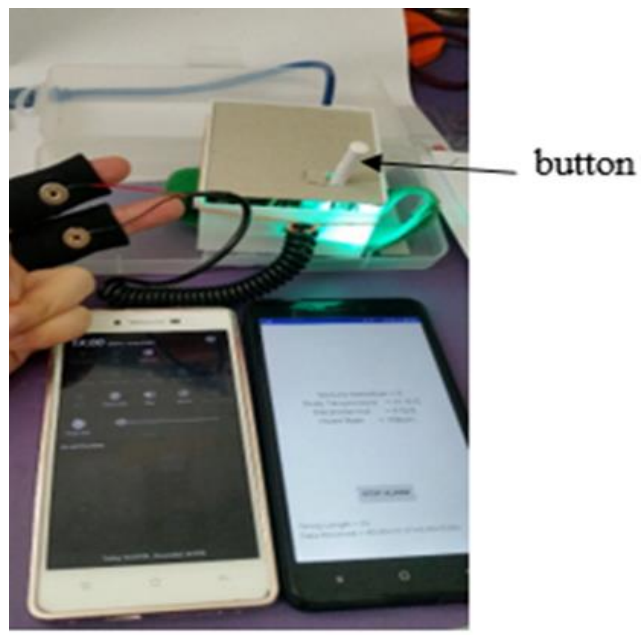

Fig. 3. Standby steps for detector function

The developed epilepsy seizure detector apps was launched and connected to the Bluetooth in the device. The personal information of patient and emergency contact number are required to fill in the developed epilepsy seizure detector apps at the first time as shown in Figure 4.

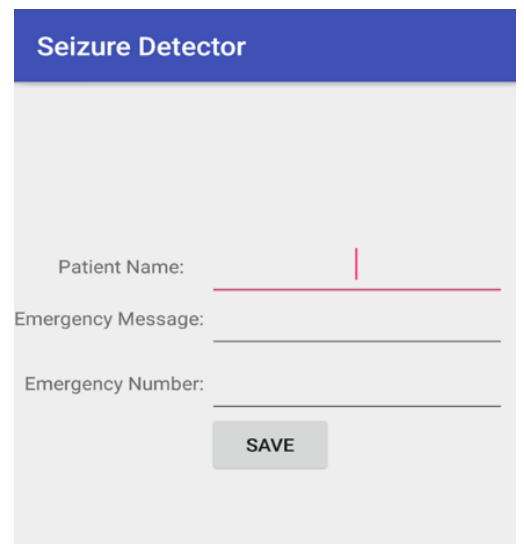

Fig. 4. Personal information page

The emergency message is will be send to the phone of number saved as seizures happen. As the two fingers equipped with electrodes were twitched, the skin conductance is changed. The RGB led implemented on the epilepsy seizures detector is turned to red colour and the alarm on the developed epilepsy seizure detector apps will be triggered at once. The alarm is used to alert the surrounding people near to the patients to give them helps promptly. The first aids knowledge to help seizures' patient will be shown by the apps through the patient's phone as in Figure 5. The condition of the patient will be saved in the Firebase database which can be observed by doctor. 


\section{Seizure Detector \\ 1. Protect patient from injury \\ Cushion patient's head with something soft if they \\ have collapsed to the ground \\ Don't hold patient down \\ Don't put anything in patient's mouth \\ 2. Gently roll the patient onto one side as soon as it \\ is practical to do so \\ Stay and calmly talk to the patient until \\ consciousness is regained \\ 3. Dial 112 for an ambulance if a shaking seizure doesn't stop after 5 minutes}

Fig. 5. First aid instruction

\section{Results \& Discussion}

As can be seen in Figure 6, before confirming the epilepsy seizure, the developed epilepsy seizure detector apps recorded that the status of "Seizure Detection" is 0 and changes to 1 to ring the bell after the confirmation.

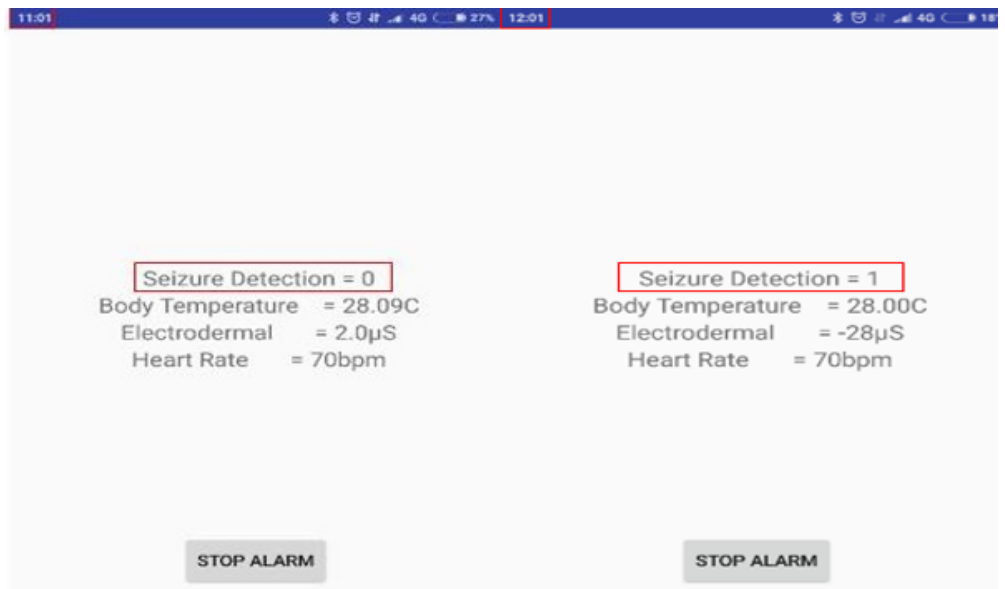

Fig. 6. Detection of epilepsy seizure

In Figure 7, RGB LED changes from green to red after seizure detected. Meanwhile, the emergency message was sent to the doctor's phone for the notification purposes, as shown in Figure 8. On the other hand, Firebase database was set to collect the data from the developed epilepsy seizure detector apps with interval of 2 seconds. 

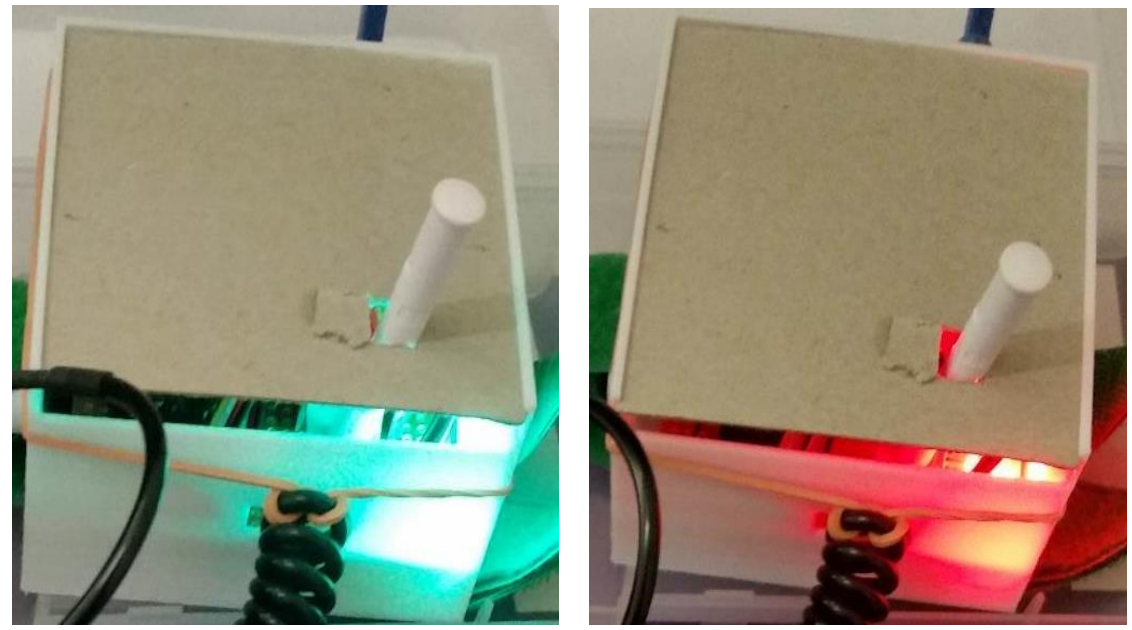

Fig. 7. RGB LED changes from green to red after detection

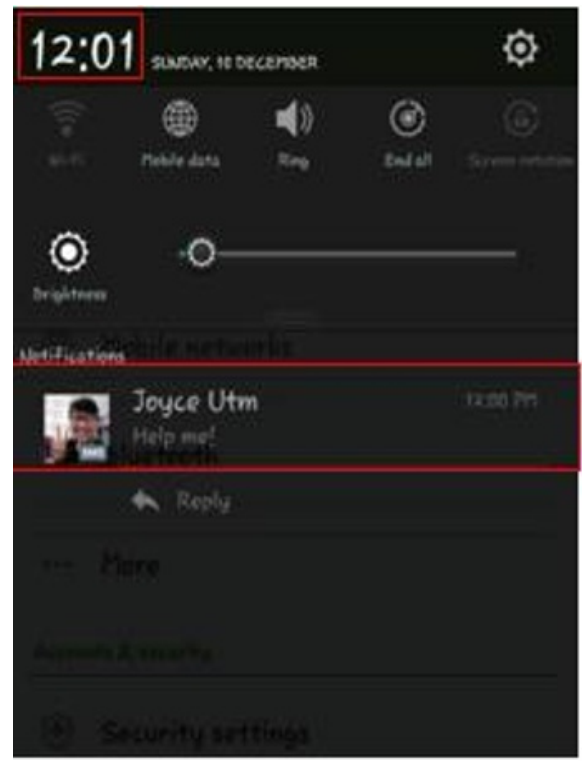

Fig. 8. The emergency message received on doctor's phone

Figure 9 shows that the data which is collected at $12.01 \mathrm{pm}$ showed that the seizure occurred while the other time showed the detection was false. 


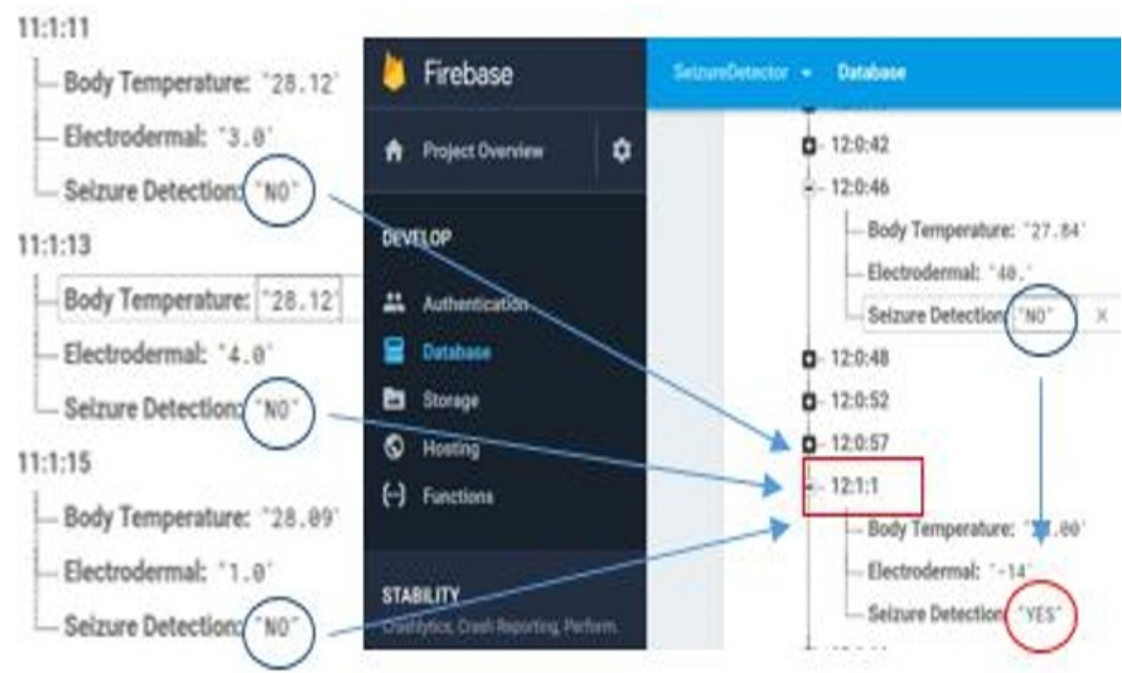

Fig. 9. Seizure detection on Firebase database

Stability test was conducted. The whole processes were conducted repeatedly for five times. Results from Firebase database, changes of RGB LED, doctor's phone and the developed epilepsy seizure detector apps were observed. Data collected after testing the device for 5 times is shown in Table 2.

Table 2. Data collected for four items to be checked for 5 repetitive test

\begin{tabular}{|c|r|r|r|r|r|}
\hline \multicolumn{1}{|c|}{ Tries } & \multicolumn{1}{c|}{$1^{\text {st }}$} & \multicolumn{1}{c|}{$2^{\text {nd }}$} & \multicolumn{1}{c|}{$3^{\text {rd }}$} & \multicolumn{1}{|c|}{${ }^{\text {th }}$} & \multicolumn{1}{|c|}{} \\
\hline RGB LED & $\checkmark$ & $\checkmark$ & $\checkmark$ & $\checkmark$ & $\checkmark$ \\
\hline $\begin{array}{c}\text { Developed epilepsy } \\
\text { seizure detector apps }\end{array}$ & $\checkmark$ & $\checkmark$ & $\checkmark$ & & $\checkmark$ \\
\hline Doctor's phone & $\checkmark$ & $\checkmark$ & $\checkmark$ & & $\checkmark$ \\
\hline Firebase database & $\checkmark$ & $\checkmark$ & $\checkmark$ & & $\checkmark$ \\
\hline
\end{tabular}

From Table 2, we can observe that the device passes 4 tests out of 5 . In other words, the device has $80 \%$ of accuracy and precision which also means that the system has $20 \%$ of possibilities on facing detection failure. Besides, from the same table, when the seizure occurred, RGB LED has $100 \%$ correct detection, meanwhile the developed epilepsy seizure detector apps might fail and causes other items (dcotor's phone and Firebase database) to fail too.

The detector fails to detect the seizure sometimes due to the contradiction of serial communication between Arduino Nano and the developed epilepsy seizure detector apps. The same problem causes the accelerometer fail to detect a fall as the delay needed to transmit the data was too long until the accelerometer fails to detect the fall on time. Somehow, the device is not said to be bad but there are some improvements needed to enhance the user experience. For instances, higher sensitivity sensors could be used to replace the one used in this project to give better results. Besides, the Bluetooth serial communication between the developed epilepsy seizure detector apps and Arduino Board 
can be improved by replacing a better Bluetooth chip. As the detection of seizure occurrence was critical in this project.

\section{Conclusion}

The epilepsy seizure detector was built with low cost materials which could meet the demand of medium-income family which is the majority group of people in the world. Moreover, the device comes along with an the developed epilepsy seizure detector apps which is user friendly and easy to be used. It helps to collect data for further analysis by doctors or health advisors, instead of sending emergency message to their family member. In short, the device is good enough to detect the seizure but somehow the accuracy is low.

\section{References}

1. Epilepsy Society, (2017, November). Seizures. An Introduction to Epileptic Seizure. Retrieved from: https://www.epilepsysociety.org.uk

2. Mayo Clinic, (2017). Epilepsy Symptoms and causes. Retrieved from https://www.mayoclinic.org/diseases-conditions/epilepsy/symptoms-causes/

3. Jon Glass, (2011, October 11). Epilepsy. Seizures disorder. Retreived from https://www.medicinenet.com/seizure/article.htm

4. WebMD Medical Reference, (2017, July 12). Types of Seizures and their Symptoms. Retrieved from https://www.webmd.com/epilepsy/ 